\title{
The role of nutrition in immunity in infants and toddlers: An expert panel opinion
}

\author{
Uday A. Pai ${ }^{a}$, Priya Chandrasekhar ${ }^{\mathrm{b}}$, Ryan S. Carvalho ${ }^{\mathrm{c}}$, Shweta Kumar ${ }^{\mathrm{d}, *}$ \\ a Department of Pediatrics, AANGAN Clinic, Mumbai, India \\ b Department of Pediatrics, Apollo Children's Hospital, Chennai, India \\ ${ }^{c}$ Medical, Scientific E' Regulatory Affairs Nestle Infant Nutrition, Nestlé, North America, USA \\ ' Medical Affairs, Medico-Marketing \& Public Health, Medical \& Scientific Affairs, Nestlé, Gurgaon, India
}

\section{A R T I C L E I N F O}

\section{Article history:}

Received 26 October 2017

Accepted 28 November 2017

Available online 29 November 2017

\section{Keywords:}

Immunity

Micronutrients

Macronutrients

Supplementation

Fortification

Dietary Diversification

Vaccination visits

\section{A B S T R A C T}

Maternal nutritional status during pregnancy and the nutrients to which infants are exposed through breastmilk and complementary foods influence infant immune system development. Micronutrients, including iron, zinc, vitamins A, C, D, and E exert important influences on immune system development during the neonatal period. An expert panel meeting was convened to provide insights on strategies to prevent and mitigate micronutrients deficiencies (MNDs) which impact the developing immune system. This review article provides evidence-based correlation between the role of nutrition and immunity, and provides recommendations on the current and future trends to prevent and treat MNDs in the Indian context.

(C) 2017 Published by Elsevier, a division of RELX India, Pvt. Ltd on behalf of INDIACLEN.

\section{Introduction}

\subsection{Immune system development: An Overview}

The immune system encompasses the innate and adaptive immunity (Table 1). ${ }^{1,2}$ The initial hematopoietic cells begin to develop in the yolk sac and thus herald the development of the immune system during embryogenesis. The thymus, which plays a central role in development of immune system, begins to develop by the 7 th week of gestation, while the bone marrow begins to develop by the 20th week. ${ }^{3,4}$ The T lymphocytes, natural killer (NK) cells and various dendritic cells (DCs) develop in the thymus, whereas the B lymphocytes, monocytes, granulocytes, and some DCs develop in the bone marrow. The lymphoid system is developed at birth, but it remains functionally immature. ${ }^{3}$

Given that the immune system of neonates is immature and not fully functional, their susceptibility to infections and ability to respond appropriately is impaired. However, neonates are protected against infections through passive immunity; conferred through IgG and IgA antibodies, transferred from the mother

\footnotetext{
* Corresponding author.

E-mail address: Shweta.Kumar@in.nestle.com (S. Kumar).
}

through the placenta during the last trimester of pregnancy, and through the breastmilk during lactation, respectively. ${ }^{3}$

\subsection{Nutrition: Role in development of neonatal immune response}

The fetal immune system is susceptible to any inflammatory, infectious, environmental, or nutritional changes during the prenatal period. ${ }^{5}$ A significant exposure or insult from any of these factors during pregnancy may significantly affect the programming of function and/or structure of the thymus which may lead to permanent alteration in the population of specific immune cells resulting in immune deficiencies. ${ }^{4}$

Studies in animal models have shown that micronutrient deficiencies (MNDs) in utero negatively influence the size and function of the thymus, alter the function of lymphocytes, and reduce the size of $\mathrm{B}$ lymphocytes. Thus, prenatal nutritional insults will have negative alterations in immune programming resulting in increased risk of infection during infancy and inflammatory diseases later in life. ${ }^{5}$ These findings highlight the importance of adequate and appropriate nutrition during the prenatal period and early years of life for immune system establishment and adequate immune response. ${ }^{6}$ 
Table 1

Innate vs. adaptive immunity.

\begin{tabular}{|c|c|}
\hline Innate immunity & Adaptive immunity \\
\hline Provides first line of defense ${ }^{1}$ & Serves as second line of defense ${ }^{1}$ \\
\hline Activated as soon as an antigen is encountered ${ }^{1}$ & Triggered for secondary elimination of infectious agents ${ }^{1}$ \\
\hline Comprises neutrophils, macrophages, monocytes, and dendritic cells ${ }^{2}$ & Comprises $\mathrm{T}$ cells and $\mathrm{B}$ cells ${ }^{1}$ \\
\hline Has no immunologic memory ${ }^{1}$ & Has immunologic memory ${ }^{1}$ \\
\hline
\end{tabular}

\section{Objectives and methods}

Given that adequate nutrition is crucial for the development of the neonatal immune system, an expert panel meeting was convened in April 2017 to discuss this topic further. An expert panel comprising key opinion leaders, pediatricians, and nutrition experts gathered to brainstorm and discuss the role of various macro and micronutrients in the neonatal immune system development. The expert panel also discussed various strategies that can be used to prevent and treat MNDs. This review article is a compilation of literature evidence and recommendations of the expert panel on the role of nutrition in development of immune system.

\section{Prenatal and postnatal nutritional status: Impact on neonatal immune response}

Maternal nutritional status from preconception through lactation plays a crucial role in programming the developing organ systems and homeostatic pathways in the offspring. The first trimester of pregnancy is characterized by rapid development and proliferation of immune system and related organs. Subsequently, the immune system continues to develop and mature during the early years of life. ${ }^{5}$

Maternal malnutrition or reduction in nutrient supply can also directly affect the immune system development of fetus by affecting placentation and thereby altering transfer of immune factors through placenta and breastmilk. A compromised fetal nutritional status affects organogenesis, growth, and fetal programming. ${ }^{6}$

The postnatal environmental insults that infants are exposed to and the nutrition they consume through breast milk, breast milk substitutes, and complementary foods play a crucial role in the development of immunity in early childhood.,7 Postnatally, nutrients exert an effect on innate immune signal transduction pathways and immune cell development, which influence early allergen sensitivity, promote tolerance towards emerging gut microbiota and ingested antigens, and patterns of host defense against pathogens. ${ }^{8}$

\subsection{Breast milk: A key factor in immune system development}

Breast milk, the first food to which neonates are exposed, serves a valuable source through which the immunological memory is transferred from the mother to the infant. ${ }^{9,10}$ It contains several biologically active, antimicrobial peptides (AMPs), like defensins and cathelicidin, and immune-modulatory compounds that support active and passive immunity during the early years of life. ${ }^{3,11}$ Cathelicidin (an AMP) is stored in inactive proform in the granules of neutrophils which proparts cathelin after neutrophil degranulation. Various functions like endotoxin neutralization, angiogenic, chemotactic and wound healing reveal cathelicidin's role as a mediator between innate and adaptive immunity. ${ }^{11}$ Breast milk components like probiotics and oligosaccharides also play a role in the developing intestinal microbiome of the infant, which is critical in immune system development. ${ }^{3}$ Evidence indicates genetic traits transferred from mother to child through breast milk composition also alters the offspring's immune response. ${ }^{9}$

\section{Malnutrition and infection: Interactive effects on immune response}

Malnutrition or undernutrition is associated with impaired immunity with consequent increased susceptibility to infections and cytokine activation. ${ }^{8}$ Deficiency of immuno-nutrients may result in impaired immune response by affecting the immune system at the cellular level, e.g., affecting cell function, mucosal barrier, etc. Studies have shown that severe malnutrition can affect the development of thymus, which consequently leads to impaired immunity. On the other hand, infection also contributes to a malnourished state. Infection triggers an array of immune system inflammatory cascades that results in further stressing the developing immune system. Consequently, the outcome of infection is determined in some parts by the nutritional status of the host. These findings indicate that malnutrition and infection exhibit a vicious bidirectional relationship. ${ }^{12}$

\section{Role of different nutrients in immune response and the consequences of their deficiencies}

\subsection{Macronutrients}

Amino acids are crucial for immune system since they regulate the activation of T lymphocytes, B lymphocytes, macrophages, and NK cells; production of cytokines and antibodies; lymphocyte proliferation; and gene expression. ${ }^{13}$ Studies suggests that carnosine (bioactive peptide found in meats and fish) has the capacity to modulate the immune system of an individual by increasing the production of interlukin- $1 \beta$ and by suppressing the apoptosis of neutrophils. ${ }^{14}$ The LC-PUFAs are essential for immune cell responses, since they contribute to membrane fluidity within cell membranes, gene expression and signal transduction, and provision of substrates for production of chemical mediators. ${ }^{15}$ Carbohydrates have a key role to play for an adequate immune response as lymphocytes, macrophages, neutrophils, and other immune cells require glucose to meet their high metabolic needs. ${ }^{16}$ Of all carbohydrates, galactose involving interactions play an important role in host defenses. All the structures containing galactose are involved in immune systems, both innate and adaptive immunity. ${ }^{17}$

\subsection{Micronutrients}

Micronutrients regulate cell mediated and innate immunity, modulate cytokine expression, humoral antibody response and play a crucial role in the differentiation and development of Th1 and Th2 lymphocyte subsets. ${ }^{8,18}$ Studies have established that micronutrients such as iron, zinc, selenium, vitamin A, C, D, and E, and folic acid are essential for efficient immune system functioning. ${ }^{18}$ Apart from these, micronutrients like copper and chromium 
are significantly important in immunomodulation. Animal models have shown that copper deficiency results in reduced $\mathrm{T}$ cell proliferation thereby decreasing humoral, cell- mediated and nonspecific immune response suggesting its role in maintenance in immune competence. ${ }^{19-21}$ Chromium, on the other hand, alters immunostimulatory or immunosuppressive processes thereby affecting immune response that causes hypersensitivity reactions; it also has an influence on T and B lymphocytes; and cytokine and macrophage production. ${ }^{21}$

Despite significant economic advancements, the nutritional status of infants and children continues to be worrying. According to the National Family Health Survey-2, -3 , and -4 reports, a significant proportion of children under the age of 3 and 5 years are stunted, wasted, and underweight (Fig. 1). Poor nutritional status is associated with MNDs. ${ }^{22-24}$

\subsection{Prebiotics}

Studies have shown that the addition of prebiotics, nondigestible oligosaccharides, in the diet of an infant may impact their risk of developing allergic diseases, by stimulating the growth and metabolism of protective commensal microbes in the infant gut. These findings support the role of dietary components in modulating immune development. ${ }^{10,16}$

\subsection{Probiotics}

Probiotics are live microorganisms that exert beneficial effects when administered in adequate quantities ${ }^{10}$ by interacting with the gut microbiota. i.e., they fortify the gut's barrier function and modulate the immune system. Probiotics also interact with cells of the immune system, especially dendritic cells, and improve regulatory $\mathrm{T}$ cell function. ${ }^{3}$

\section{Strategies to prevent and treat micronutrient deficiencies}

The United Nations Children's Fund supports various strategies for the prevention and treatment of MNDs in women and children. $^{25}$

\subsection{Preventive strategies:}

\subsubsection{Dietary diversification}

Dietary diversification or modification mainly involves increasing the consumption of a variety of foods from different food groups so as to provide a balance of nutrients in the diet. Diet of infants should be both nutrient dense as well as energy dense, as making food calorie dense increases the bioavailability of nutrients in the body. ${ }^{26}$ The Indian Council of Medical Research also recommends the intake of diversified diets. ${ }^{27}$
In infants, exclusive breastfeeding should be promoted during the initial 6 months of life, and continued up to 2 years or beyond. ${ }^{28}$ Breast feeding remains the best way to support nutrition and the developing immune system in an ideal way for the mother-infant dyad. ${ }^{29}$ Furthermore, it is important to ensure that complementary foods provided after 6 months of age are not only diverse but also rich in micronutrients. ${ }^{28}$ In most developing countries, however, the consistency of complementary foods prepared using cereals, or tubers and starchy roots is insufficient (i.e. thin or watery) to meet the developmental and nutritional needs. Further, these grains inherently contain anti nutrients like tannins or phytates which inhibit the absorption of certain micronutrients from the food. ${ }^{26}$ In India, only $54.9 \%$ of infants below 6 months of age are exclusively breastfed, while less than $50 \%$ of infants aged between $6-8$ months receive solid or semi-solid foods with breast milk. Additionally, only $9.6 \%$ of children aged $6-23$ months receive adequate diet during the complementary feeding period (Fig. 2). ${ }^{23,24}$ Therefore, caregivers should be educated on the importance of adopting household food preparation strategies (e.g. soaking, germination, and fermentation) during the preparation of cereal-based complementary foods to enhance the bioavailability of micronutrients from these foods. ${ }^{26}$

Although dietary diversification is the most sustainable and desirable strategy to prevent MNDs, lack of resources in developing countries may hamper the availability or consumption of micronutrient-rich foods. Additionally, some cultural/religious beliefs may also serve as a barrier for the consumption of animal-source foods, thereby restricting the nutritional intake. ${ }^{26,28}$

\subsubsection{Fortification}

Given that universal access to adequate food is a major factor limiting the adoption of appropriate diverse dietary habits, food fortification offers the dual advantage of providing nutrients to large segments of population without necessitating any changes in food consumption patterns. ${ }^{28}$ Food fortification is also costeffective, compared to other strategies, and regular consumption of fortified foods helps to maintain steady body stores of the micronutrients. ${ }^{30}$

Fortification programs are increasingly being considered as an effective approach to combat malnutrition in developing countries. The Government of India has recommended food fortification in the 10th, 11th, and 12th Five-Year Plan as a strategy to improve nutritional status through existing government nutritional programs. $^{31}$

The World Health Organization recommends that complementary foods be rich in iron or fortified with iron-containing micronutrient powders to enhance iron status and decrease the incidence of iron deficiency anemia in populations where anemia prevalence in children aged $<2-5$ years is $\geq 20 \%{ }^{32} \mathrm{~A}$ study by Detzel has demonstrated that in India, the current consumption of

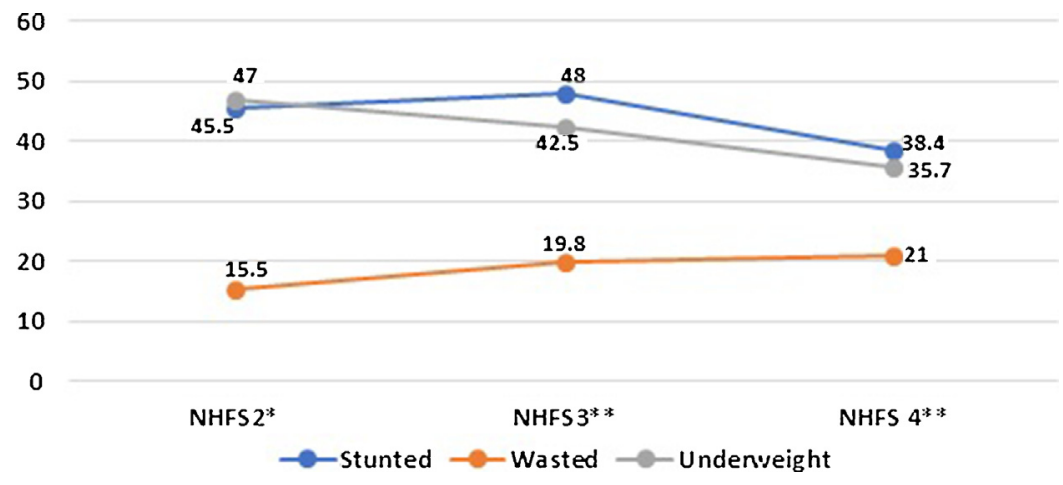

Fig. 1. Nutritional status of infants in India. ${ }^{22-24}$ 


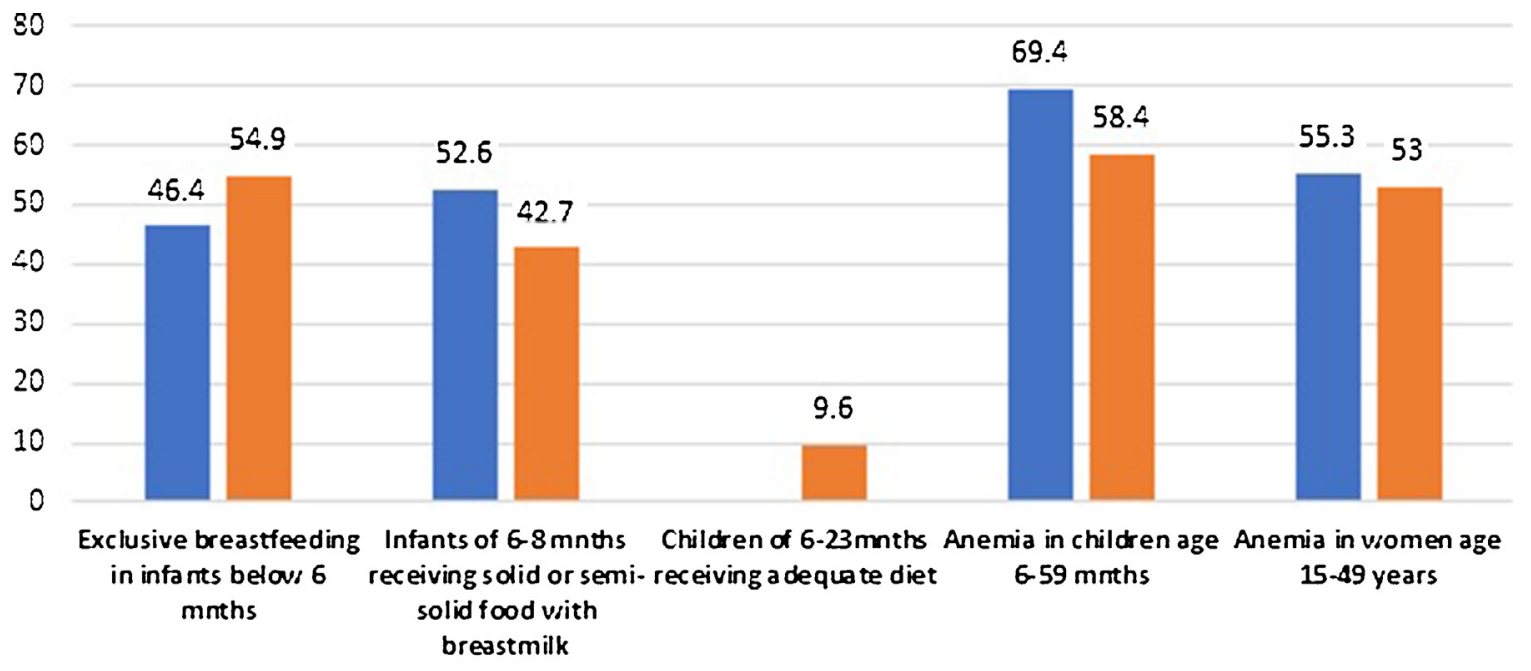

NFHS-3 NFHS-

Fig. 2. Nutritional status of infants and children, and child feeding practices in India. ${ }^{23,24}$

fortified infant foods (e.g. fortified cereals) has already reduced the annual iron deficiency anemia burden, representing a 50\% reduction in the health burden (in terms of disability-adjusted life years) and a $36 \%$ reduction in economic losses. ${ }^{33}$

\subsection{Therapeutic strategy}

\subsubsection{Supplementation}

Micronutrient supplementation serves as a therapeutic strategy to deliver micronutrients that are crucial for growth and development, in relatively large doses in the form of syrups or pills, to pregnant women and children. ${ }^{28,34}$

The Indian government has implemented several national supplementation programs including the Iron-Folic Acid supplementation and National Iron Plus Initiative. ${ }^{35}$ However, research indicates that only $13.8 \%$ of children aged 6-59 months benefited from the Iron-Folic Acid supplementation program. ${ }^{36}$

Although supplementation is an appropriate tool to prevent MNDs, it requires an exhaustive supply chain process, a commitment to compliance and may not be sustainable for large populations with widespread deficiencies. ${ }^{37}$ Further, it is a preferred strategy when clinically diagnosed severe micronutrient deficiency needs therapeutic intervention and should be used only as a short-term strategy. ${ }^{34}$ Poor compliance and lack of supplies are the major barriers to supplementation programs. ${ }^{28}$

Evidence indicates that dietary diversification and food fortification are more effective than supplementation. ${ }^{34}$

\section{Nutrients and Immunity: Current Trends and Way Forward}

The immune system is a structurally complex and functionally diverse organ system that is influenced by nutrition among other factors. The immunity of an individual depends on both qualitative as well as quantitative aspects of immune response. The developing fetal and neonatal immune system can be affected by genetic predisposition, maternal stress, maternal nutritional status, exposure to environmental contaminants, infant's nutritional adequacy (quantitative levels macro \& micronutrients) etc.

Despite the fact that the neonatal immune system is vulnerable to insults during the critical window of development, owing to various factors, and has a multitude of biomarkers and proteomic markers but there is no single test that can predict the immune status of an infant. The duration, severity \& repeated episodes of infections in a child may indicate a weak immune system which may be due to the presence of undernutrition as well as micronutrient deficiency and other associated factors. Thus, addressing hidden hunger at an early stage may help an infant to acquire their maximum growth \& development potential. Hence measurement of key immune supporting-nutrients like iron, zinc, vitamin B12, and vitamin D levels may be beneficial to determine a deficiency state. Iron which is categorized as a micronutrient is evolving in importance to a macronutrient because of the key role it plays in immune system, hematopoietic system, growth and cognitive development in infants and toddlers. Serum ferritin levels can be evaluated to determine the need for iron supplementation and hemoglobin levels can be used as a marker to detect iron deficiency anemia

Predicting an impaired immune system clinically, poses a challenge as there are no fixed set of symptoms to gauge it. Close follow up on a child's growth chart may serve as one of the markers available clinically to assess macronutrient deficiency. However, growth chart may not be valuable to detect micronutrient deficiency, except in severe deficiency states. In general, if a child is marching along a growth chart in its proper percentile, it may be a reliable indicator for sufficient macronutrients in the diet. To be sure of the overall health \& well-being of an infant, a balanced nutrition with required amount of macro \& micro-nutrients should be provided from the time of initiation of complementary feeding.

The expert panel concluded that there is huge need of awareness to parents or guardians for role of nutrition in first 1000 days of an infant. This role of nutrition does not pertain only to the growth \& development of an infant but also to the immune system programming. Any nutritional inadequacy or nutritional insult during this period may have long term consequences like impaired immune system, cognition etc. resulting in increased susceptibility to infections or even reduced 5-10 IQ points later in life.

The expert panel recommends increased general nutritional awareness amongst care givers, parents and guardians. The approach of medical fraternity should be to offer age- specific, practical, problem-solving education and nutritional counselling on feeding practices for an infant. In current Indian scenario, where public health problems, like iron deficiency anemia, is prevalent, knowledge implementation of nutritional counselling becomes critical. Medical fraternity can help achieve it by targeting various touch points like vaccination visits, routine check-up visits or other 
visits to maximize the opportunities to emphasize the benefits of nutrition through counselling so as to improve dietary adequacy, growth \& health of an infant and help them to achieve their maximum potential of growth and development.

\section{Conflict of Interest}

Authors, UAP, PC, none to declare.

Authors RSC and SK are employees of Nestlé North America and India Respectively.

\section{Funding}

The program is funded by Nestlé Nutrition Institute, South Asia.

\section{Acknowledgements}

We would like to acknowledge BioQuest Solutions for their support in editorial services.

\section{References}

1. Warrington R, Watson W, Kim HL, Antonetti FR. An introduction to immunology and immunopathology. Allergy Asthma Clin Immunol. 2011;7:S1.

[2]. Simon AK, Hollander GA, McMichael A. Evolution of the immune system in humans from infancy to old age. Proc R Soc B. 2017;82:20143085.

3. Niers L, Stasse-Wolthius M, Rombouts FM, Rijkers GT. Nutritional support for the infant's immune system. Nutrition Rev. 2007;65:347-360.

4. Moore SE, Fulford AJC, Wagatsuma Y, Persson LÅ, Arifeen SE, Prentice AM. Thymus development and infant and child mortality in rural Bangladesh. Int J Epidemiol. 2014;43:216-223.

5. Palmer AC. Nutritionally mediated programming of the developing immune system. Adv Nutr. 2011;2:377-395.

6. Marques AH, O'Connor TG, Roth C, Susser E, Bjørke-Monsen AL. The influence of maternal prenatal and early childhood nutrition and maternal prenatal stress on offspring immune system development and neurodevelopmental disorders. Front Neurosci. 2013;7:120.

7. Jain N, Walker WA. Diet and host-microbial crosstalk in postnatal intestinal immune homeostasis. Nat Rev Gastroenterol Hepatol. 2015;12:14-25.

8. Cunningham-Rundles S, Lin H, Ho-Lin D, Dnistrian A, Cassileth BR, Perlman JM. Role of nutrients in the development of neonatal immune response. Nutr Rev. 2009;67:S152-S163.

9. M'Rabet L, Vos AP, Boehm G, Garssen J. Breast-feeding and its role in early development of the immune system in infants: consequences for health late in life. J Nutr. 2008;138:1782S-1790S.

[10]. Nauta AJ, Ben Amor K, Knol J, Garssen J, van der Beek EM. Relevance of pre- and postnatal nutrition to development and interplay between the microbiota and metabolic and immune systems. Am J Clin Nutr. 2013;98:586S-593S.

11. Kai-Larsen Ylva. The Role of the multifunctional peptide ll-37 in host defense. Front Biosci. 2008;13:3760-3767.

12. Schaible UE, Kaufmann SH. Malnutrition and infection: complex mechanisms and global impacts. PLoS Med. 2007;4:e115.

13. Li P, Yi YL, Li D, Kim SW, Wu G. Amino acids and immune function. Br J Nutr. 2007;98:237-252.

14. Tan KM, Candlish Carnosine JK. Anserine as modulators of neutrophil function. Clin Lab Haematol. 1998;20:239-244.
15. Dubai J. The role of nutritional status in immunity of infants and young children. IJSR. 2013;4:473-475.

16. Gleeson M, Bishop NC. Modification of immune responses to exercise by carbohydrate, glutamine and anti-oxidant supplements. Immunol Cell Biol. 2000;78:554-561.

17. Brockhausen I. The Role of Galactosyltransferases in Cell Surface Functions and in the Immune System. Drug News Perspect. 2006;19:401-409.

18. Maggini S, Wintergerst ES, Beveridge S, Hornig DH. Selected vitamins and trace elements support immune function by strengthening epithelial barriers and cellular and humoral immune responses. Br J Nutr. 2007;98:S29-35.

19. Stabel JR, Spears JW. Effect of copper on immune function and disease resistance. Adv Exp Med Biol. 1989;258:243-252.

20. Percival SS. Copper and Immunity. Am J Clin Nutr. 1998;67:1064S-1068S.

21. Shrivastava R, Upreti RK, Seth PK, Chaturvedi UC. Effects of Chromium on the Immune System. FEMS Immunol Med Microbiol. 2002;34:1-7.

22. NFHS-2 fact sheet. . Available at: Accessed on: 13 July, 2017 http://rchiips.org/ nfhs/data/india/keyfact.pdf.

23. National family health survey (NFHS-3) India 2005-06. . Available at: Accessed on: 13 July 2017. http://rchiips.org/nfhs/ nutrition_report_for_website_18sep09.pdf.

24. National family health survey-4, 2015-16. . Available at: Accessed on: 13 July 2017 http://rchiips.org/NFHS/pdf/NFHS4/India.pdf.

25. Micronutrients. UNICEF; 2017. Available at: Accessed on June 2, 2017. https:// www.unicef.org/nutrition/index_iodine.html.

26. Zlotkin S, Tondeur M. Specific strategies to address micronutrient deficiencies in the young child: supplementation and home fortification. Pettifor JM, Zlotkin S, eds. Micronutrient deficiencies during the weaning period and the first years of life. nestlé nutrition workshop series pediatric program. 2004;54:233248.

27. Indian council of medical research. Dietary guidelines for Indians. A manual. . Available at Accessed on June 2, 2017. http://ninindia.org/ dietaryguidelinesforninwebsite.pdf.

[28]. World Health Organization guidelines on food fortification with micronutrients. . Available at: Accessed on June 2 2017. http://www.who.int/nutrition/ publications/guide_food_fortification_micronutrients.pdf.

29. Walters D, Kakietek JJ, Eberwein JD, Pullum T, Shekar M. Breastfeeding in the 21 St Century. Lancet. 2016;387(10033):2087.

30. Das JK, Salam RA, Kumar R, Bhutta ZA. Micronutrient fortification of food and its impact on woman and child health: a systematic review. Syst Rev. 2013;2:67.

31. Liu P, Bhatia R, Pachon H, Emory MD. Food fortification in India: A literature review. Indian J Comm Health. 2014;26:59-74.

32. Multiple micronutrient powders for point-of-use fortification of foods consumed by infants and children. World Health Organization; 2017. Available at: Accessed on 16 May 2017. http://www.who.int/elena/titles/ guidance_summaries/micronutrientpowder_infants/en/.

33. Detzel P. estimating the contribution on the reduction of the burden of iron deficiency anemia from the current fortification strategy for commercial foods targeted to infants and toddlers in India. .

34. Dairo MD, Ige OK. Supplementation of micronutrient in community micronutrient deficiency prevention programmes. Ann Ib Postgrad Med. 2009;7:6-9.

35. Deb S. Emplimentation of national iron plus initiative for child health: challanges ahead. Indian J Public Health. 2015;59:1-2.

[36]. Ministry of women and child development government of india. rapid survey on children. 2013-2014. India fact sheet. :2. Available at: Accessed on: 06 June 2017. http://wcd.nic.in/issnip/National_Fact\%20sheet_RSOC\%20_02-07-2015. pdf.

[37]. Berry J, Mukherjee P, Shastry G. Taken with a grain of salt? Micronutrient fortification in South Asia. . Available at: Accessed on 16 May 2017. http:// academics.wellesley.edu/Economics/gshastry/berry\%20mukherjee\% 20shastry\%202012\%20fortification.pdf. 